\title{
Computer-Mediated Communication(CMC) as a tool for language teaching
}

\author{
Mustafa Khalid Saleh Al-Rawi \\ MAEnglish Language and Applied Linguistics (ELAL) \\ Assistant Lecturer at the department of English Language, Cihan University - Sulaimanyah -Iraq
}

\section{1.INTRODUCTION}

mediatedcommunication(CMC)isthemostsignificantandpopularmilieuthatvelopthehumanlife.Itcanbedefi nedasthe(human)communicationthatoccursthroughtheuseofcomputersortechnicaldevices;asMetz(1994citedinMah di,2014:9)putsit"anycommunicationpatternmediatedthroughthecomputer".Itisusedtocommunicateindividualsovert heworldprovidingthemwithopportunitiestoshareinformationsignificantlylanguage, witheachother.Particularly,(whi chisalsothefocusofthisessay)thedevelopmentofthetechnicalcommunicationprovidesopportunitiesforlanguageteach ingandlearning.Thesetypesofopportunitiesarenotactuallyexistedinthetraditional'(conventional)classrooms.Origina lly,communicationsystemswerenotusedforhumantohumancommunication;theywereusedtosenddata(Murray,2005) .Thesystems, then, developedtoincludethe 'humanhumancommunication'.Therefore, thenetworkingsystemshavemo vedfrominformationtechnology(IT)toincludecommunicationwithinit(aftertheinventionofemails), soitbecameinfor mationandcommunicationtechnology(ICT)(Ibid,2005).Therearetwomodesofhumancommunication:theasynchrono uscommunicationwhichisthenonsimultaneouscommunication,forinstance:emails, discussionresponsesandonlinepo sts.Thesecondmodeisthesimultaneouscommunication,forinstance:chatandinstantmessages.Itisworthtomentionthat; communicationsystemsarenotentirelyachievedthroughcomputersorothertechnicaldevices.Internetconnectionisanin tegralpartofthecommunication.ThereforeThorne(2008)definedCMCasamultimodalsystemthatparticipateswith 'inte rnetmediatedcommunication'.AccordingtoCastells(2004citedinThorne,2008), thedailyactivitiesaretransformedinto theeducational,professionalandinterpersonalworldbytheuseofinternet.However,accessinginternetcontinuestobeneq uallydistributedtowardssocialsclassesand 'geopoliticalregions'(Thorne,2008). AlthoughCMCariseswithinternet,itis alsoarisingfrommediatedcommunicationstechnologies-forexample:television,telephone,printing

pressaswellasradioTherearealargenumberofstudiesregardingCMC,becauseitisimportanttounderstanditsro leindevelopinglife.Moreover,itisimportanttounderstandandevaluateCMCasasystemconsideringlanguageteachinga ndlearning.Thisessaywouldreviewthefindingsofanumberofcomputermediatedcommunication'srecentstudiesregard ing:theenvironmentsofCMC.Then,thesefindingwouldbelinkedtoagroupofArab(Iraqi)learnersofEnglishInaddition,t hecharacteristicsofthisgroupandanumberofshortcomingsofCMCregarding thiscontextwouldbementioned.

\section{SYNCHRONOUSANDASYNCHRONOUS}

Asmentionedabovethesynchronousmodeisthesimultaneousandtheasynchronousmodeisthenonmultaneous .Thereareanumberofresearchesregardingbothmodes.Thisessay wouldshowanumberofresearchfindings:

\subsection{Synchronous}

Intermsofsynchronousmode,AsterhanandEisenmann(2011)didastudyexploringthepreferencesandexperie ncesofsecondaryschoolstudents. Theysimplycompareddifferentformatsofdiscussion:facetofaceandsynchronousCM C.Theydividedstudentsintotwogroups:studentswhoareactiveinfacetofacediscussionsand'silent'students.Theyfoun doutthatthereareseveraladvantagesofthesynchronousCMCdiscussionformatespeciallyin 'socialinteractive' and 'man agerialaspects'oftheclassdiscussions.Moreover,silentstudentwouldpreferthesynchronousCMCincomparisonwithth eface-to-facemodeofdiscussion.

\subsection{Asynchronous}

SevingilandBayyurta(2010)examinedtheinfluenceofasynchronousmodeofcommunicationontheperceptio nsofTurkishnonnativespeakers(NNSs)ofEnglish.Byexchangingonlineemailmessages(inEnglish)withnativespeaker sofEnglishandnonnativespeakersofothercountries,(theyfound)TurkishNNSsofEnglishbenefitedfromtheseemailsin gaining 'culturalinformation' anddevelopingforeignlanguagelearningTherefore, learnersencouragedtheuseofasynch ronousCMC,becauseithelpedthem understandingthe targetlanguage. 


\subsection{Synchronousvs.Asynchronous}

Hrastinski(2008)didacomparativestudybetweensynchronousandasynchronousmodesandfoundoutthatsyn chronousonlinediscussionsprompted 'personalparticipation'(whichincreasestheinteraction)whereasasynchronouso nlinediscussionsencouraged 'cognitiveparticipation'(whichprovidesamorereflectivetypeofparticipation).Therefore, thestudentsinsynchronousdiscussionssensedthattheyareworkingtogetherandalsotheyarenotrestrictedtothecourseco ntent.HsiehandJi(2013)didacomparativestudyoftheeffectsofthreemodesofcommunication:synchronousandasynchr onousonlinecommunication, andtraditionalgrammartranslationmethod,onagroupofEFLlearners. Theydividethemint othreegroups,andthenmadetheexperiment.Theyfoundoutthattherewasnoconsiderabledifferenceinthereadingscores betweensynchronousandasynchronousgroups.Moreover,intheir(groups)'perceptionsurvey',therewerenostatisticald ifferencesbetweenthemOztoketal.(2013)inspectedtherelationshipsbetweenlearners'useofsynchronousprivatemessa gesandtheiruseofasynchronousdiscussionforums. Theyfoundoutthatasynchronousdiscussionforumscontainmorefor mal(academic)languageandlessinformal(social)language, whilesynchronousprivatemessagesarethecontrarylessfor malandmoreinformallanguage.Inaddition, theyfoundthatlearnerswhousesynchronousprivatemessagesarelesslikelyt oquicklyreadthediscussionforumsandthefactthattheyspendmoretimeonlinethanthosewhodonotusesynchronouspriv atemessages.Therefore, theysuggestedusingasynchronousdiscussionsforthosewhousemoresynchronousprivate messages.

\section{FACEBOOK,SKYPEANDYOUTUBE}

Facebook,SkypeandYouTubeare,nowadays,thenewenvironmentsofCMC.Theyhelptocommunicatepeople overtheworldbydifferentways:writtenwork,audioandvideo.Theyareusedincomputersandothertechnicaldevices,suc has:smartphones.Astheyareubiquitousinmostoftheworld,itispossibletoapplytheminthefieldoflanguageteachingandl earning.Byapplyingthem,teaching/learningwouldbeappliedoutsideclassroomsMoreover, thatwouldincreasethemult iculturalaspectofteachingorlearningi.e.teachersfromdifferentpartsoftheworldwouldteachlearnersfromotherparts(di stanceteachinglearning).Itisworthtomentionthatthereareanumberofdifferencesintheusageofeachonetheseenvironm ents.TherearealargenumberofstudiesregardingtheseenvironmentsThisessaywouldshowanumberofstudiesfindingre gardingeachoneofthem.

\subsection{Facebook}

Facebookisoneofthemostfamoussocialnetworkingwebsites.Ithasalargenumberofusersfromallovertheworl d.Ithelpstoconnectusersinasynchronousmodeofcommunicationaswellassynchronousmode(Ifbothindividualsareonl inethereisanindicationifauserisonlineandrecently,onecouldknowwhethertheindividuals/heiscontactingsawhis/herm essageornot).Allwaysofcommunication(written, audio,video)arepossibletousewhichcreatesalargeropportunitytosha reinformation.Inlanguageteaching/learningcontext,Facebookofferspossibleatmosphereofteaching/learninginthesen sethatusingitwouldfostertheinteractionbetweenteachersandlearnersandlearnersthemselvesInthestudyofKabilan, $\mathrm{Ah}$ mad,andAbidin,(2010),theytriedtofindoutwhethertheuniversitylearnersregardFacebookasasuitableenvironmentforl anguagelearningthatouldhelp,promoteanddeveloptheirlearningofEnglish. Theyfoundoutthatlearners 'believed'thatF acebookcouldbeusedasasuitable'online'environmenttodeveloptheirEnglishlanguagelearning. Yet,teachershaveasig nificantroleinintegratingandutilisingFacebookforeducationalpurposes.Mitchell(2012)examinedthemotivationoflea rnersforusingFacebookwhichismostlyusedforsocialpurposes. Theyuseitforaperiodoftimeandthentheywereinterview ed.Theresultsshowedthattheyhadagreatabilityinusingittocommunicatewiththeirexistingfriends, learnEnglish,andlea rnaboutfriends'cultures. However, therewereaslightnumberofdifficulties consideringits use.

\subsection{Skype}

Skypeisarogrammethatprovidestextmessagingaswellasvideoandaudiochat.Itiswidelyusedacademically,be causeofitsabilityofconferencingcallsi.e.morethantwoindividualcandoanaudiochattingatthesametime.Becauseofitsa bilities,onecouldassumethatitcouldbeasignificantlybeneficialtoolforlanguageteaching/learning(Mahdi,2014).Inthe studyofDevelotte, GuichonandVincent(2010), theyfoundthatusingSkypeassynchronousmode(videochat)wouldimpr ovetheinteractionbetweenlearnersandteachers.However,thelessuseofwebcam(video)might,toagreatextent,effectthe interactionthereasonseemstoberelatedtocognitiveaspectsoflanguage.Yanguas(2010)researchedtheuseofaudioandvi deoSkypechattingbylearnerswhiledoingataskbasedinteraction.HefoundthattheturntakingwhileusingSkypeisconsid erablyclosetothefacetofaceturntaking.Therefore, itispossibletoassumethatmostlearnerswouldnotdisagreewithusing Skypeacademically,regardlesstheshortcomingsofCMCthatwouldbe mentionedlater.

\subsection{YouTube}

YouTubeisawebsite.Itisusedforvideosharing.Individualscanview,uploadandsharevideos.Itcontainsaveryl argenumberofvariostypesofvideos.Lectures,educationalandculturalinformationcanbeeasilyuploaded.Therefore, Yo uTubecouldbeusedforlanguagelearning.Itissimplylikeattendinglecturesanywhereatanytime.YouTubeisacommunic ationthroughvideos; however,thereisaspaceofpostswhichissimplyappliedforpostingopinionsaboutwhathasbeenseen .Thisspacecouldbeusedacademicallyasaformofasynchronousonlinediscussion.Miller,HafnerandFun(2012)foundou 
that,learnersofEnglishforacademicpurposes(EAP)courseunderstoodthevalues(linguisticallyandtechnically)ofcons tructingandsharingvideosonYouTube.

\section{WIKI}

Awikiisanunlockedwebsiteinthesensethatitisfreelyaccessibleandnotonlyauthoritativeuserscanadd,edit,ord eleteitscontent.Anyuser,however,canadd,edit,ordeletecontenteasily.Wikicanhavelicensecontroloption,so,itcanbere strictedtoanydding,editingordeleting,justasMoodle.Therefore,thereisaconsiderablepossibilityofwikispacestobeuse dforeducationalpurposes, especiallycollaborativewritinginlanguagelearning.AccordingtoKesslerandBikowski(201 0:43), "theevolutionofcollaborativewritingmaybeintrinsicallyconnectedwiththeiterationsoftechnologysincenewdev elopmentsprovidenewopportunitiesforcollaboration".Nottoforgetthatwikispacestendtolackauthoritativenessandreli ability,becauseoftheabilityofanyonetochange,addordelete.KesslerandBikowski(2010)studiedtheeffectsofwikibase dcollaborativeactivityonthebehaviourofEFLlearners. Theyfoundoutthatusingflexiblelearningenvironments(collabo rativeautonomouslanguagelearningandCMCwikibasedcollaborativeactivity)wouldbenefitthelearners.Inaddition,e veniflearnersdonotconsistentlyusewikispacesinthewaytheyareinstructedto, wikiisbeneficial,becauseitcreatesaproce ssofengaginglearnerstowritetogether.Li(2013)exploredtheeffectofwikispacesonChineseEFLuniversitylearners.Th efocuswasonwikibasedcollaborativewriting.Theresearcherfoundthattheengagementoflearnerswassignificantnotonl yinthediscussioncontentbutalsointaskmanagement,socialchat,languagenegotiationandtechnicalcommunication.Fur thermore, thelearnershave'scaffolded'oneanother'swritingwhileconstructingthewiki.Itispossibletosaythattheuseof wikiwouldimprovethe learners'collaboration.

\section{FEEDBACK}

Intermsoffeedback,computermediatedcommunication(CMC)studieshaveshownthatCMC'sfeedbackappe arstobeeffectiveinlanguagelearning.BuenoAlastuey(2013)studiedtheinteractionalfeedbackandparticularlyLanguag eRelatedEpisodes(LREs)whichmeansthepartsofinteractionswhenalearnershiftsfrommeaningtoform. Theresearcher didastudyofthreegroupsoflearners(nonnativeswiththesamefirstlanguageL1, nonnativeswithdifferentL1andnonnativ eswithnatives)toseewhetherinteractingwithdifferentpartnershasaneffectonthenumberandtypeofLREsbyusingSync hronousVoicebasedComputerMediatedCommunication. Theresearcherfoundoutthat:LREsfocusonmeaningandfor $\mathrm{m}$,interactingwithdifferentpartnerhasconsiderablyinfluencedthetypeandthenumberofLREsandtherewereinsightsof negativefeedbackandnegotiation. Therefore, thequalityandquantityoffeedbackwoulddependmostlyoninterpersonala spects.GurzynskiWeissandBaralt(2014)investigatedtheperceptionoflanguagelearnersandtheiruseoffeedbackinatas kbasedinteraction.Itisacomparativestudybetweentwomodesofcommunication:CMCandfacetofaceinteraction.They foundthatinbothmodes, learnersnoticedfeedbackasafeedback.Inbothmodesofcommunication,perceptionaccuracyof feedbackhasthesamestatisticalrate.However,thelearners'chancestousefeedbackaredependingmostlyonthetypeofint eractionaswell asthe erroraddressed.

\section{CULTURALAWARENESS}

Cultureinthiscasemeans'stereotype'whichcouldbedefinedastheclassificationofdifferentaspectaccordingto individuals'cognitiveprocesses(Houghton,etal.2013)Becauseoftherapidspreadoflanguagelearningovertheworld,cul tureappearedtobeconsideredasanobstacle.Therefore,criticalculturalawarenesswasapplied;whichmeans “anabilityto evaluate,criticallyandonthebasisofexplicitcriteria,perspectives,practicesandproductsinone' sownandotherculturesa ndcountries"(Byram,1997citedinHoughtonetal.2013:1).Still,stereotypeswouldhaveaninfluenceoverlearning.Wu, MarekandChen(2013)studiedtheculturalawarenessofEFLlearners, whileprovidingthemwithculturaldifferencesands imilaritiescourseusingCMCInternetvideoconferences. Theircriticalthinkingwasanalysedandrevealedthathistoryand cultureareimportant,theirviewofmulticulturalworldisimprovingandfinally,suchacourseincreasestheirconfidenceFol lowingHall'sculturaldimensionofcontextuality,Pflug(2011)analysedGermansandIndianspostingsininternetforums. TheresearcherfoundoutthatGermansshowedlessculturalcontextthanIndians.Moreover,Indiansutilisedmore"emotic ons'thanGermansandthisuse(ofnonverbalcommunication)suggestsrevealingculturalcontext.Therefore,culture/soci al aspectsseemtohaveinfluence on CMC.

\section{GENDER}

ThereappearnottobealargenumberofCMCstudiesregardinggender.However,itcouldbeapossibleissuethatin fluenceCMCespeciallyontheinteractionalprocesses.GuillerandDurndell(2007)didastudyonthelanguageuseinasync hronouscomputermediatedcommunication(CMC),specifically,todistinguishtheroleofgenderingroupdiscussions $\mathrm{Th}$ eyfoundoutthatbothgendersutilisedsimilarlinguisticvariables, butfemalesused 'intensifiers'morethanmales.Malesus ed'authoritativelanguage'and'negativeinteractions'whilefemalesusedmorepersonalandemotionalcontributions. Th ereforegenderappearstobeasignificantfactorindiscussionsandwouldhaveaneffectonCMCinlanguagelearning.Cubuk cuandKutlu(2013)researchedtheCMC'sattitudesregardinggenderin(synchronousandasynchronous)chat,andalsothe influenceofgenderonndividual'slinguisticchoiceswhenchatting. TheyfoundoutthatCMCissomehowsimilartoface- 
to-facecommunicationinthesensethattheyarebothputanumberofcommunicationrestrictionsonparticipants andtheyareusingthe same strategyofturn-taking.

\section{CHARACTERISTICSOFTHEGROUP}

Theyareauniversitymonolingualgroupconsistingofapproximately(3545)learners.Theteachingprocessistak ingplaceinclassroomswhicharenotwelltechnicallyequipped.TheircoursecanbeconsideredasgeneralEnglishlanguage .Thegroupcanbedividedintofivesubgroupsintermsoftheirintelligenceandtechnicalunderstandingi.e.theproficiencyin usingcomputersandothertechnicaldevices.(a)Learnerswhocouldbeperfectinlearningprocesswhatevercontexttheyar eini.e.theywouldnotbeaffectedwhenmovingfromthetraditionalclassroomstowardsthecomputerisedlearningenviron mentorviceversa.(b)Learnerswhoareperfectintermsofintelligenceandtechnicalunderstanding,butstilltheymighthave problemswithmovingfromonesystemtoanother.(c)Learnerswhoareperfectintermsofintelligence, buttheyhavedifficu ltyinusingdevelopedtechnology.(d)Learnerswhoareweakofinsufficientintermsofintelligence, however, theycouldbe perfectinusingdevelopedtechnology.Finally,(e)Learnerswhoareinsufficientinboth:intelligenceandtechnology.Thes edivisionsareappliedandjudgedaccordingtoexperience, asteachingthegroupfortwoyearswouldprovidemoreinformat ion.Moreover,knowingthelearnersforalongperiodoftimewouldgivetheteacherexpectationsoftheirreactiontowardsu nfamiliarconditionsthattheymayencounter.Thereasonbehindthisdivisionisthatitmightgiveaclearerpictureowardsthe influenceofCMC, andonecouldunderstandtheadvantagesanddisadvantagesofthesystem(CMC)easily(aswellasitwou ldhelpforfurtherCMCresearchthere).Forexample:learnerfromgroup(a)wouldnotactuallygiveanyinformationofwhet herusingCMCisbetterthanusingthetraditionalway,becausesimplys/heseemstobeperfectinboth,thenhis/herpositivevi ewwouldbebalanced.Thesamecasewithalearnerfromgroup(e)his/hernegativeviewtowardsCMCwouldalsobenegati vetowardsthetraditionalwayHowever,motivationandlearners'beliefstendtoaffecttheirCMCjudgement.Inaddition,th egroups(b,candd)arethosewhomightprovideaclearerjudgementofCMCbutalsomotivationandlearnersbeliefsplayasi gnificantroleintheirjudgementsmotivationinthissenseiseithercognitive(intrinsic), individualwhoisnotwillingtolearn Englishlanguage,orsocial(extrinsic), anindividualwhothinkthatEnglishisnotbeneficialtohim/herors/hehastolearnino rdertopassexamsoracourse.Moreover,cognitiveandsocioculturalaspectswouldaffectthelearners'judgements.Finally ,itisworthmentioningthatknowingthe participantsofthe studypersonally appear toinfluencethe findingsofthe research.

\section{LIMITATIONSREGARDINGTHEGROUPS'USEOFCMC}

Thereareactuallyanumberoflimitationsregardingthecontextoftheselectedgroupoflearners. Thefirstandmost importantlimitationisthatCMC,aslanguagelearningenvironment,hasrarely(ifever)beenusedinIraqyet.Nostudiesapp eartobefoundinusingCMCforlanguageteaching/learning.Therefore, thereflectionoflearners'useofCMCwouldbeass umedaccordingtoexperience.However,CMCisbeingrecentlyusedforsocialpurposes. Thesecondproblemisaccessibili ty.Accessibilityinthissensecanbedividedintohreemainaspects:thetechnologicalavailability, theinternetaccessibilitya ndthelicensedpagesorprogrammes.Intermsoftechnologicalavailability, anumberoflearnersdonothavecomputers, oth ersdohavebuttheycannotusethemappropriately.Althoughlargenumberoflearnershasandusescomputersorothertechni caldevicesandthefactthattechnologyisrapidlydeveloping,smallnumberoflearnerswholackthetechnicaluseandaccessi bilitywouldmakeaninfluenceonusingCMCinclassrooms.Moreover,thegroups'universitydohavealanguagelaborator $\mathrm{y}$ (soundlab)andcontains2030computerswhicharenotenoughforalllearnersatatime.Asfortheinternetaccessibility,inte rnetseemstobeprovidedmostlyeverywhereinIraq,however, theserviceappearstobenotofaconsiderablequalityInterms oflicensedpagesandprogrammes, thegroups'universitydoesnotprovidetheirlearnerswithsoftwarelicensesi.e.thepaidf or(notfree)websitesandprogrammes. Therefore, materialswouldbeutoftheir(learners)hands. Thethirdissueistheperce ptionsandbeliefsofteachersandlearners, whichmeans, anumberofteachersaswellaslearnerswouldassumethatthetraditi onalwayofteachingandlearningisbetterthantheCMCenvironmentsoflearning.Inaddition,itcouldbepossibleiflearners orteachersperceivecomputersorothertechnicaldevicesdifferentlyi.e.theymaythinkofthemasnotaconsiderableenviro nmentofeducation.

\section{THEGROUP'SASSUMEDREFLECTIONSTO WARDSTHEMENTIONEDSTUDIES}

Accordingtosynchronousandasynchronousmodes,bothmethodsareconsiderablybeneficialinlanguagelearn ing.However,eachoneofthemhasanumberofaspectsthatlearnersmayfindthemusefulornotdependingonthetypeandthe subjectofinteraction.InthecontextoftheIraqilearners, theywouldpreferthesynchronousmodewhentheyareinteracting witheachotherbecauseasfoundinOztoketal.(2012)study,synchronousmodetendstobemoreinformalandsociableasito ffersinstantsent/receivedreplies. Whereaswhileinteractingwithteachers, theypreferasynchronousmodeofcommunica tionasitismoreformalthansynchronous.Moreover,itisdistancingteachersfromlearnersi.e.Iraqilearnerstendnottobestr onglyrelatedtotheirteachersbecauseofculturalissuesofrespecteveniftherearerelations, thentheywouldbewithcertainli mits, butthatdoesnotmeantheteachersareunfriendly,itisjustasputtingtheteachersonahigherstatusoutofrespectIndditio $\mathrm{n}$,asynchronousmodewouldgivelearnersmoretimetochecktheirresponsesbeforeinteractingwithteachers; (theymostly seemtobeafraidofcommittinganygrammaticalorspellingmistakes)FacebookSkypeand YouTubeareknownbyalmosta 
lllearners, becauseoftheirspreadovertheworld.Theymightstronglywelcometheideaofusingthoseenvironmentsinlang uagelearningastheseenvironmentsprovidesignificantopportunitiestocommunicate(bytextsaudiosandvideos)withpe oplefromdifferentlanguagesandcultures.However,teachersandanumberoflearnerswouldconsiderthoseenvironment sastimeconsuming;theymaytemptearnersandmovetheirattentionandfocuswhilelearning.Furthermore,theseenviron mentscanberegardedunreliableandunauthoritative,forthefacthatanyonecanwrite,speakorrecordavideo.Thiscouldno tbe,however, alimitationbecauseteacherscancontroldiscussions, videosandtalkeasily.Wikiswouldalsobeencouraged bybothteachersandlearners.ItwouldbepossibletosuggestWikisforonlinereadingtoIraqilearners, justasanattempttoco mmunicatelanguagewithtechnology(computers/otherdevices). Therefore, thisessaywouldsuggestthatwhenapplyingt heseenvironmentswiththeselectedIraqigrouponlinereadingwouldbetakenasafirststeptowardsimplementingtechnolo gywithlanguagelearning.Readinginthissenseisbothcontrolled(structuredreadingactivities)andfree(extensivereading Intermsoffeedback,Iraqilearnersmay,toagreatextent,preferteachers'feedback; theytendnottoacceptpeers'feedbackb ecausetheyprobablywantthefeedbackfromsomeonehoisinahigherlevel(alsotheyafraidiftheirmistakesrevealedinfron toftheirpeers).However,peerfeedbackisalsoexistedbutitdependsontherelationshipofpeersasbothresearchesaboverev ealed.Moreover,itwouldbebetterforteachers/learnersiftheyuseasynchronousinteractionalfeedback, becauseitisprivat eandcouldhappenatanytimeandanywhere, theyalsowouldpreferfacetofaceteachers'feedback, becauseitmayprobably openthedoorsthataresomehowrestrictedinasynchronousmodeofdiscussionsandestions.Intermsofculturalawareness, culturewouldstronglyinfluenceCMCinperceptionsofbothteachersandlearners. ThustheybothwouldencourageCMCl anguageteaching/learningenvironments, becausetheymightshareculturalknowledgeandunderstandingfromllpartsoft heworld.Asforgenders,probablytheresultsofthefindingofbothresearcheswouldbethesameinthecontextofIraqilearner s.Moreover, thetechnicalandinternetaccessibilityoffemalesappearstobeslightlylessthanmales,so,malescouldbeablet ousetechnicaldevicesmore.However,accordingtotheselectedIraqigroupoflearners,femalescanbeconsideredmoresup portiveandencouragingthanmalesinCMC environmentsforlanguagelearning.

\section{CONCLUSION}

Computer-

mediatedcommunicationisrapidlydeveloping.Lifeintherecenteradependsmoreontechnology.Ascomputeri sedcommunicationisutilisedinalmosteveryfieldoflifeitcanbeutilisedinlanguagelearning.Largenumberofstudiesreve aledtheprivilegesofimplementingCMConlanguagelearning.Furthermore, thereisapossibilitythatCMClearningwoul dtakeoverthetraditionalwayofclassroomlearningbecauseofthebenefitsandvariableenvironmentsthatCMCoffers.Acc ordingtoallthefindingrevealedinthisessay,CMCenvironmentswouldbesignificantlybeneficialinlanguagelearningast heyprovidealargenumberofopportunitiesofcommunication,collaboration, andlanguageandculturalknowledge.Inthe contextoftheIraqigroupoflearners, itwouldbealsobeneficialaswellasdevelopmentalimplementingCMCenvironments foreducationalpurposesespeciallylanguagelearning.However,largenumberoflearnersbecauseofthelimitationsmenti onedabovewouldfinddifficultiesusingCMC.Therefore,CMCmightbeappliedasablendedcoursewiththetraditionalcla ssrooms. Themaintainedlearners'reflectionsarelimitedasthereseemtobenopreviousstudiestosupportclaims(claimsar ebasedonexperience). Furtherstudywouldprovideauthenticinvestigativeresearch.

\section{REFERENCES}

[1] Asterhan,C.S.andEisenmann,T.(2011).Introducingsynchronousediscussiontoolsincolocatedclassrooms:Ast udyonthe experiencesof 'active'and'silent'

[2] secondaryschoolstudents.ComputersinHumanBehavior,27(6),2169-2177.Bueno-

Alastuey,M.C.(2013).InteractionalfeedbackinSynchronousVoicebasedComputerMediatedCommunication: Effectofdyad.System,41(3), 543-559.

[3] Çubukçu,H.andKutlu,Ö.(2013).ComputerMediatedCommunication:AnObservationonGenderinChatRooms .Procedia-SocialandBehavioralSciences, 70, 724-730.

[4] Develotte,C.Guichon,N.andVincent,C.(2010)theuseofwebcamforteachingaforeignlanguageinadesktopvide oconferencingenvironment.ReCALL, 22(3), 293-312.

[5] Guiller,J.andDurndell,A.(2007).Students'linguisticbehaviourinonlinediscussiongroups:Doesgendermatter? .ComputersinHumanBehavior,23(5), 2240-2255.

[6] GurzynskiWeiss,L.andBaralt,M.(2014).Exploringlearnerperceptionanduseoftaskbasedinteractionalfeedbac kinFTFandCMCmodes.StudiesinSecondLanguageAcquisition, 36,1-37.

[7] Houghton,S.A.Furumura,Y.Lebedko,MandLi,S.(eds.)(2013)CriticalCulturalAwareness:Managingstereoty pesthroughIntercultural(language)Education, UK:Cambridge Scholars publishing.

[8] Hrastinski,S.(2008). The potentialofsynchronous communicationtoenhance participationinonline discussions:Acase studyoftwo e-learningcourses. Information\&Management,45(7), 499-506.

[9] Hsieh,P. C.andJi,C.H. (2013). The EffectsofComputer-MediatedCommunication bya Course ManagementSystem(MOODLE)On EnglishReadingAchievementand Perceptions.201-205.

[10] Kabilan,M.K.,Ahmad,N.andAbidin,M.J.Z.(2010).FacebookAnonlineenvironmentforlearningofEnglishinin stitutionsofhighereducation?.TheInternetandHigherEducation, 13(4),179-187. 
[11] Kessler,G.andBikowski,D.(2010).Developingcollaborativeautonomouslearningbilitiesincomputermediated languagelearning:attentiontomeaningamong studentsinwikispace.ComputerAssistedLanguageLearning,23(1),4158Li,M.(2013)Individualnovicesandcol lective experts:Collective scaffoldingin

[12] wiki-basedsmallgroupwriting.System,41(3),752-

769Mahdi,H.S.(2014).TheImpactofComputerMediatedCommunicationEnvironmentsonForeignLanguageL earning:AReviewofthe Literature.WorldJournalofEnglishLanguage,4(1),p9.

[13] Miller,L.Hafner,C.A.andFun,C.N.K.(2012)Projectbasedlearninginatechnologicallyenhancedlearningenviro nmentforsecondlanguagelearners: Students'perceptions.E-LearningandDigitalMedia,9(2), 183-195.

[14] Mitchell,K.(2012).Asocialtool:WhyandhowESOL students use Facebook. CALICOJournal,29(3),471-493.

[15] Murray,D.E.(2005)Newfrontiersintechnologyandteaching,inDavison,C. InformationTechnologyandInnovationinLanguageEducation, HongKong:Hong KongUniversityPress.

[16] Oztok, M., ZingaroD.,Brett,C.andHewitt,J.(2013).Exploringasynchronousand synchronoustoolusingonline courses.Computers\&Education,60(1),87-94.

[17] Pflug,J.(2011).Contextualityandcomputermediatedcommunication:acrossculturalcomparison.Computersin HumanBehavior,27(1),131-137.

[18] Sevingil,E.andBayyurt,Y.(2010).MakingashifttowardsnewCMCmodes.ProcediaSocialandBehavioralScien ces,2(2),2980-2984.

[19] Thorne,S.L.(2008).Computer-MediatedCommunication.InEncyclopediaoflanguageandeducation(2ndEd.)4 ,325-336.

[20] Wu,W.C.V.,Marek,M.andChen,N.S.(2013).AssessingculturalawarenessandlinguisticcompetencyofEFLlea rnersina CMC-basedactivelearningcontext. System,41(3),515-528.

[21] Yanguas,I.(2010).OralcomputermediatedinteractionbetweenL2learners:It'sabouttime!LanguageLearning\& Technology,14(3), 72-93. 